\title{
The Results of Balanced Orbital Volume Increase Surgery With Porous Polyethylene (Medpor) Implants After Post- Traumatic Enophthalmos: Case Reports
}

\author{
Selam Yekta Sendul ${ }^{\mathrm{a}, \mathrm{b}}$, Burcu Dirimª, Zeynep Acar ${ }^{\mathrm{a}}$, Mehmet Demir ${ }^{\mathrm{a}}$, Dilek Guven ${ }^{\mathrm{a}}$
}

\begin{abstract}
Post-traumatic enophthalmos is one of the late-term complications of the posttraumatic orbit, causing functional and cosmetic concern. In this study, the process, results, efficacy and reliability of the treatment of two young cases with late-term enophthalmos after blunt trauma to the orbit without any vision loss will be presented and discussed. Surgery for a balanced orbital volume increase was performed using porous polyethylene (Medpor) implants.
\end{abstract}

Keywords: Post-traumatic enophthalmos; Treatment of enophthalmos

\section{Introduction}

Post-traumatic enophthalmos is the backward migration of the bulbus oculi in the orbital structure after trauma. The basic development mechanism is the decrease in the volume of the orbital content in the enlarging orbital bone tissue [1]. There are two major causes. The most significant one is the posttraumatic enlargement of the bone volume as a result of lack of treatment of the orbital bone structure or reconstruction of it with improper and insufficient materials. The other cause is the decrease of the orbital volume in consequence of the late-onset orbital fat tissue atrophy caused by trauma [2]. Retraction of the bulbus oculi causes the superior eyelid sulcus to deepen and cosmetic problems such as ptosis to occur. Post-traumatic enophthalmos up to $2 \mathrm{~mm}$ is acceptable functionally and esthetically, whereas cases with enophthalmos more than $2 \mathrm{~mm}$ may cause functional and esthetic issues depending on the severity of the damage [1]. Surgical treatment is inevitable when distinct enophthalmos occurs. The study was conducted in accordance with the tenets of the Declaration of Helsinki by ob-

Manuscript accepted for publication October 22, 2015

aDepartment of Ophthalmology, Sisli Hamidiye Etfal Training and Research Hospital, Istanbul, Turkey

${ }^{b}$ Corresponding Author: Selam Yekta Sendul, Department of Ophthalmology, Sisli Hamidiye Etfal Training and Research Hospital, Etfal Street 34371 Sisli, Istanbul, Turkey. Email: sysendul@hotmail.com

doi: http://dx.doi.org/10.14740/jmc2354w taining written consent from patient, with the approval of the local ethical review board. In addition, written consent of the patients for the publication of the photographs was obtained.

In this study, the process, results, efficacy and reliability of the treatment of two young cases with late-term enophthalmos after blunt trauma to the orbit will be presented and discussed. Surgery for a balanced orbital volume increase was performed using porous polyethylene (Medpor) implants.

\section{Case Reports}

\section{Case 1}

A 38-year-old male patient who had a history of inferior orbital wall fracture repairment 7 years ago caused by blunt trauma to the orbit, applied to our clinic with complaints of the eye getting smaller. Ophthalmological examination showed that the visual sharpness was complete and the anterior and posterior segments were normal. The patient who had a $4 \mathrm{~mm}$ enophthalmos spotted with Hertel exophthalmometer showed ptosis of the superior palpebra and retraction of the inferior palpebra. One implant for each medial and lateral orbital wall of the subperiosteal region and two implants for the inferior lateral orbital wall with a thickness of $3 \mathrm{~mm}$ and width of $1.5 \times 3 \mathrm{~cm}$ were placed via transconjunctival inferior orbitotomy approach. Although full recovery was observed with Hertel exophthalmometer evaluation in the post-operative month 3 , ptosis and inferior palpebral retraction still remained. In response, firstly the ptosis was treated by performing superior palpebral levator procedure and then the inferior palpebral retraction was treated by using ear-cartilage graft in the posterior lamellar region (Fig. 1). We have been continuing the follow-up for the patient for 22 months who has had no complaints other than minimal scleral show. In addition, no complications have been observed such as shift in Medpor implant, infection, damage in periocular muscles or limited globe movements.

\section{Case 2}

A 32-year-old female patient applied to our clinic complaining about the right eye getting smaller after a blunt trauma 10 years ago. Ophthalmological examination showed that the vis- 

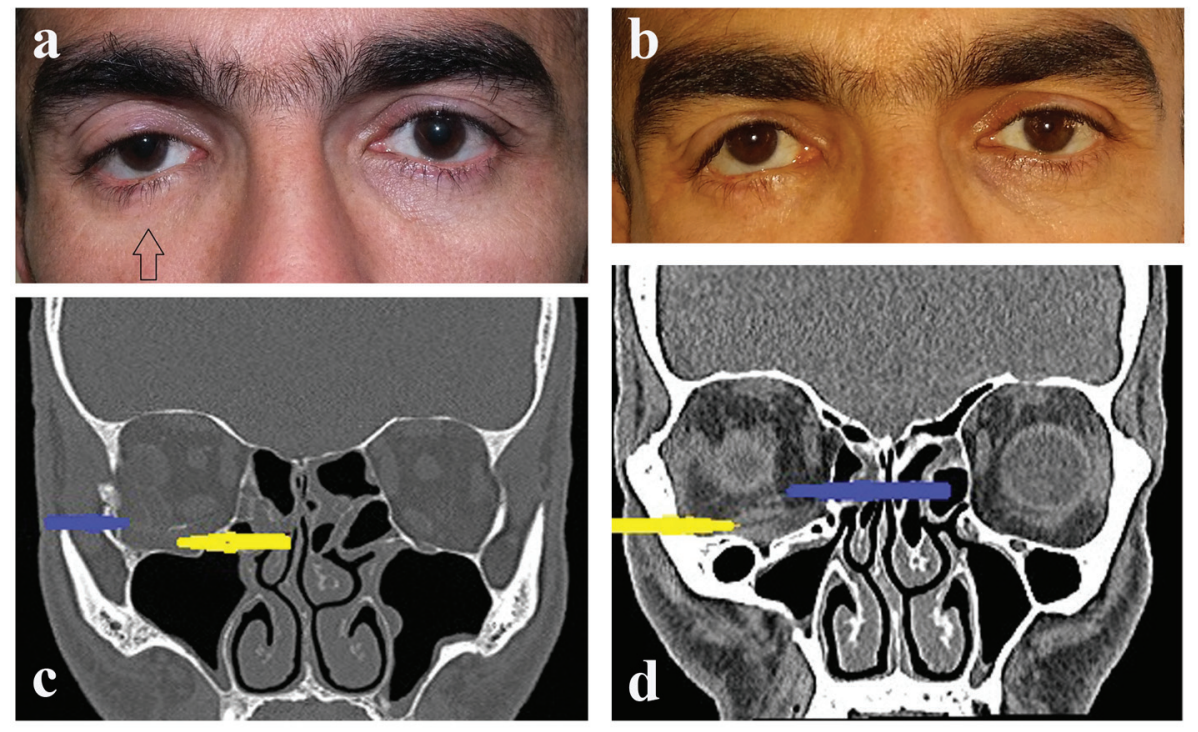

Figure 1. (a) A 38-year-old male patient. Preoperative ptosis, enophthalmos, inferior palpebral retraction with inferior fatty tissue atrophy (arrow) can be observed in the right eye. (b) Image in the post-operative year 1 after enophthalmos surgery, treatment of ptosis and inferior palpebra reconstruction with ear cartilage (slight scleral show is observed). (c) CT image of Medpor implants in the post-operative month 6 (yellow arrow: inferior, blue arrow: lateral). Volume enlargement in the orbital walls is obvious. (d) CT scan for soft tissue examination (yellow arrow: two Medpor implants may be seen in the lower orbita; blue arrow: inferior rectus muscle).

ual sharpness was complete and the anterior and posterior segments were normal. Examination with Hertel exophthalmometer showed a $3 \mathrm{~mm}$ enophthalmos and ptosis in the superior palpebra. One implant for each of medial, lateral and inferior orbital walls of the subperiosteal region with a thickness of 3 $\mathrm{mm}$ and width of $1.5 \times 2.5 \mathrm{~cm}$ was placed via transconjunctival inferior orbitotomy approach. Although full recovery was observed with Hertel exophthalmometer evaluation in the post-operative month 3 , ptosis still remained. In response, ptosis surgery was performed via levator procedure (Fig. 2). We have been continuing the follow-up of this patient for 18 months who has had no complaints. In addition, no complications have been observed such as shift in implant, infection and limited globe movements.

\section{Discussion}

Orbital blunt trauma may cause orbital bone fracture and damage on internal and peripheral orbital soft tissues [3]. Various materials are used in the surgical treatment of bone fractures after orbital blunt trauma. Autologous bone grafts, cartilagelike materials or alloplasts made of titanium, Teflon, porous polyethylene or Marlex mesh may be used as implant materials [4]. Among these materials, autologous implants have significant advantages such as successful adaptation with the tissue, revascularization, resistance to extorsion and absence of infection. Nevertheless, despite revascularization, these autologous materials can be resorbed and may cause unpredictable change in dimension, volume and eventually the position of bulbus oculi in the orbit [1]. Bones with higher dense cortical structure proportion such as calvarial bones are more resistant to resorp- tion whereas bones with higher cancellous proportion such as the ilium and costa are more vulnerable to resorption [1]. Experimental studies show that bones with higher proportion of cortical structure such as calvarial bones are less vascularized and less vulnerable to osteoclast activity compared to cancellous bones [5]. One of the major concerns about autologous implants is the difficulty of manipulating the shape of these materials perioperatively. For this reason, alloplastic materials such as Medpor have lately stood out as they are easily shaped and highly vascularized as a result of the porous complex. Extorsion and risk of infection are surely the major concerns about this material against surgical use. In a 4-year study including 70 cases with orbital fractures treated with Medpor, none of the cases showed any post-operative infection and extorsion complications [1].

Treatment of enophthalmos, which is one of the late-onset symptoms of orbital blunt trauma, is relatively difficult and problematic. The severity of the trauma and the region affected are important in the prognosis and treatment of enophthalmos. Treatment of cases with more than one orbital fracture may not be possible with these materials alone. Use of rigid metallic materials is beneficial for preparing an orbital bone frame; furthermore these materials serve as a platform for bone grafts and alloplastic implants [6]. A study on late-onset post-traumatic enophthalmos reports a $65 \%$ increase in the orbital volume and a treatment rate of $50 \%$ in severe enophthalmos cases by using custom designed titanium mesh implants created with computerized tomography (CT) images of the patients. On the other hand, the need for an additional correction for increase in orbital ingredients is indicated in the study [7].

Although it is accepted that the enlargement of the orbital bone walls is the main culprit in the pathogenesis of late-term 

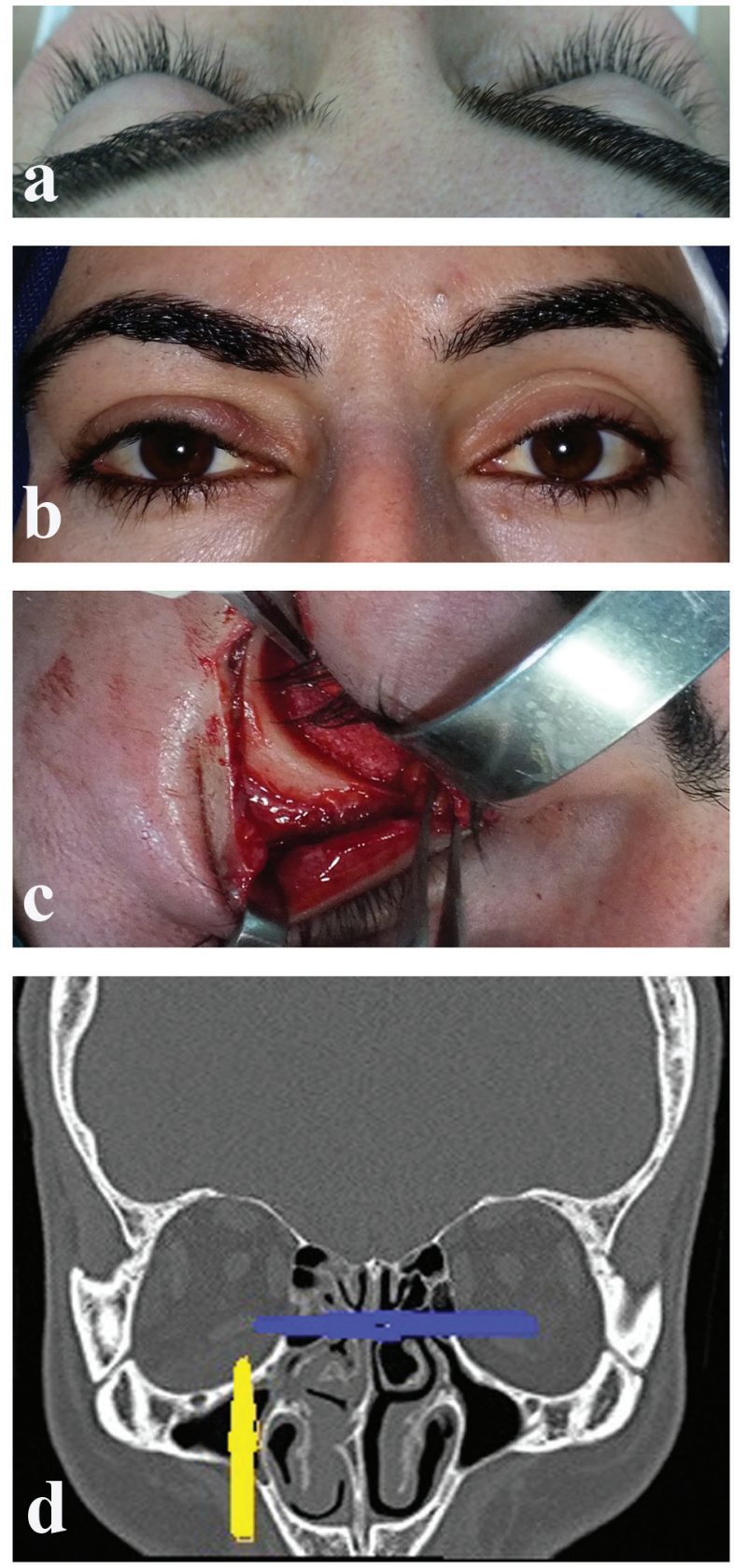

Figure 2. (a) A 32-year-old female patient with enophthalmos in the right eye. (b) Image in post-operative month 1 after enophthalmos and ptosis surgery. (c) Medpor implant can be observed on the inferior orbital wall perioperatively. (d) CT image in the post-operative month 3 after enophthalmos surgery (yellow arrow: inferior implant; blue arrow: inferior rectus muscle). The patient had intraorbital fat tissue atrophy, but no enlargement in the orbital bone volume.

enophthalmos, lately many authors have been preferring the method of increasing the orbital or periorbital soft tissues for fixing the deformities or preventing enophthalmos [8-12]. Nishi et al [9] used costal cartilage sliced from posterior to equator in the subperiosteal plan, while Lee [10] preferred using cartilage after corrective osteotomies in order to increase the volume. Cervelli et al [11] indicated in their one-case study that retrobulbar fat tissue injection is a reliable and alternate method for the treatment of enophthalmos. Honda et al [12] used tissue expander for the treatment of an enucleated enophthalmos case.

In order to provide that the bulbus oculi stands in the right anatomic position, regular orbital structure, periocular soft tissue and active periocular muscles are needed. For this reason, in the treatment of post-traumatic enophthalmos, these structures should be considered and as a matter of fact, the main objective should be adjusting damaged anatomic structures. In our surgical method, we successfully brought the bulbus oculi to the front steadily for the first time by placing Medpor implants to all three walls of the orbit. Although in our surgical method, we used more than one Medpor implant for each of the three orbital walls, neither of the patients had extorsion, strabismus or infection complications.

As a conclusion, we for the first time achieved a steady volume increase in the enophthalmic eye by using Medpor implants, without causing any vision loss. Medpor implants are alternate to autologous implants due to advantages such as vascularization and easy manipulation abilities.

\section{Conflict of Interest}

None of the authors has conflict of interest with the submission.

\section{Financial Support}

No financial support was received for this submission.

\section{References}

1. Hazani R, Yaremchuk MJ. Correction of posttraumatic enophthalmos. Arch Plast Surg. 2012;39(1):11-17.

2. Cole P, Kaufman Y, Hollier L. Principles of facial trauma: orbital fracture management. J Craniofac Surg. 2009;20(1):101-104.

3. Joseph JM, Glavas IP. Orbital fractures: a review. Clin Ophthalmol. 2011;5:95-100.

4. Mok D, Lessard L, Cordoba C, Harris PG, Nikolis A. A review of materials currently used in orbital floor reconstruction. Can J Plast Surg. 2004;12(3):134-140.

5. Chen NT, Glowacki J, Bucky LP, Hong HZ, Kim WK, Yaremchuk MJ. The roles of revascularization and resorption on endurance of craniofacial onlay bone grafts in the rabbit. Plast Reconstr Surg. 1994;93(4):714-722; discussion 723-714.

6. Glassman RD, Manson PN, Vanderkolk CA, Iliff NT, Yaremchuk MJ, Petty P, Defresne CR, et al. Rigid fixation of internal orbital fractures. Plast Reconstr Surg. 1990;86(6):1103-1109; discussion 1110-1101.

7. Zhang Y, He Y, Zhang ZY, An JG. Evaluation of the application of computer-aided shape-adapted fabricated ti- 
tanium mesh for mirroring-reconstructing orbital walls in cases of late post-traumatic enophthalmos. J Oral Maxillofac Surg. 2010;68(9):2070-2075.

8. Manson PN. Facial fractures. In: Mathes SJ, editor. Plastic surgery. Philadelphia, PA: Elsevier; 2006. p. 285-286.

9. Nishi Y, Kiyokawa K, Watanabe K, Rikimaru H, Yamauchi T. A surgical treatment of severe late posttraumatic enophthalmos using sliced costal cartilage chip grafts. J Craniofac Surg. 2006;17(4):673-679.
10. Lee JW. Treatment of enophthalmos using corrective osteotomy with concomitant cartilage-graft implantation. J Plast Reconstr Aesthet Surg. 2010;63(1):42-53.

11. Cervelli D, Gasparini G, Moro A, Grussu F, Boniello R, Pelo S. Retrobulbar lipofilling to correct the enophthalmos. J Craniofac Surg. 2011;22(5):1918-1922.

12. Honda T, Sakurai H, Nakazawa H, Isago T, Nozaki M. Correction of late post-traumatic enophthalmos using a tissue expander. Ann Plast Surg. 2006;56(6):686-688. 